
\title{
Reserarch Sulure \\ Bayesian Multilevel Analysis of Children Vaccination Coverage in Ethiopia
}

Cheru Atsmegiorgis Kitabo ( $\square$ cherueden@yahoo.com )

Hawassa University https://orcid.org/0000-0002-1713-7666

\section{Adugna Bekele}

Wachamo University

\section{Research}

Keywords: Vaccination coverage, Immunization, Bayesian multilevel analysis, Ethiopian Demographic and Health Survey, Markov chain Monte-Carlo

Posted Date: October 25th, 2021

DOl: https://doi.org/10.21203/rs.3.rs-960334/v1

License: (1) This work is licensed under a Creative Commons Attribution 4.0 International License. Read Full License 


\section{BAYESIAN MULTILEVEL ANALYSIS OF CHILDREN VACCINATION COVERAGE} IN ETHIOPIA

4

${ }^{1}$ Department of Statistics, College of Natural and Computational Sciences, Hawassa University, Hawassa, Ethiopia 


\section{Abstract}

27 Background: Vaccine preventable diseases (VPDs) account for $17 \%$ of the global under-five mortality per annum and more than half of these deaths have occurred in sub-Saharan Africa. Ethiopia is one of the ten countries which account for about $62 \%$ of unprotected children and one of the three countries (Ethiopia, Nigeria, and the Democratic Republic of Congo) in which half of the world's child deaths have occurred are in sub-Saharan Africa. The main objective of this

32 study is to understand the current status of complete immunization coverage and examine its 33 determinant factors among children in Ethiopia.

Method: Bayesian multilevel logistic regression models have been utilized to realize the 35 objectives of the research. The dataset used for this study comes from the 2016 Ethiopian Demographic and Health Survey (EDHS). The convergences of parameters are checked by using

37 Markov chain Monte-Carlo (MCMC) using SPSS and MLwiN software.

Results: The descriptive result revealed that, out of the 1929 children who are supposed to complete all basic childhood vaccines, 699 (36.2\%) children were completely vaccinated, while 1230 (63.8\%) children were incompletely vaccinated. Moreover, regions Afar, Somali and Gambela have the least proportion of vaccination coverage. Among the multilevel models,

42 Bayesian random coefficient model is found to be a better model to estimate the vaccination coverage of children. Using this model, it has been found that factors like place of residence, maternal educational, mother occupation type, Antenatal Care (ANC) utilization, Postnatal Care (PNC) utilization, type of pregnancy, household wealth index, and field worker visit were found to be the significant factors that influences the vaccination coverage.

47 Conclusion: In general, it has been estimated that the vaccination coverage in the country is relatively low and there was significant variation in the level of vaccination coverage in regions of the country.

Key words: Vaccination coverage, Immunization, Bayesian multilevel analysis, Ethiopian Demographic and Health Survey, Markov chain Monte-Carlo 


\section{Background}

55 Vaccination is the administration of a vaccine using a biological substance intended to stimulate

56 a recipient's immune system to produce antibodies or undergo other changes that provide future

57 protection against specific infectious diseases [1].

58 Vaccination is the stimulation of changes in the immune system through which that protection

59 occurs. It is one of the prevention strategies for common childhood illnesses. It prevents

60 morbidities and mortalities from diphtheria, hepatitis B, measles, mumps, pertussis, pneumonia,

61 polio, rotavirus diarrhea, rubella, cervical cancer, and tetanus. It is also one of the most powerful

62 and cost-effective of all health interventions. It prevents debilitating illness and disability and

63 saves millions of lives every year. Vaccination is also a key to achieve the Millennium

64 Development Goals (MDGs) commitments made by world leaders in 2000 to reduce poverty and

65 improve human development. The contribution of immunization is especially critical to

66 achieving the goal to reduce deaths among children under five years old (MDG 4).Vaccines have

67 the power not only to save, but also to transform lives giving children a chance to grow up

68 healthy, go to school, and improve their life prospects [2].Vaccines protect the future health of

69 all populations.

70 Universal immunization of children against six common vaccine-preventable diseases, namely

71 tuberculosis, diphtheria, whooping cough (pertussis), tetanus, polio, and measles, is crucial in

72 reducing infant and child mortality. Other childhood vaccines given in Ethiopia protect against

73 hepatitis B, and Haemophilus influenzae type b (Hib). The government of Ethiopia introduced

74 the pneumococcal conjugate vaccine (PCV 13) and monovalent human rotavirus vaccine (RV1)

75 into the nation's infant immunization programme in November 2011 and October 2012,

76 respectively. The pneumococcal vaccine protects against Streptococcus pneumoniae bacteria,

77 which cause severe pneumonia, meningitis, and otherillnesses. Rotavirus causes gastroenteritis,

78 an inflammation of the stomach and intestines. If left untreated, it can lead to severe dehydration

79 and death [3].

80 Vaccine Preventable Diseases (VPDs) account for 17\% of the global under-five mortality per

81 annum. In 2019, 5.2 million children died, and about 14,000 children still die every day 82 worldwide. Children continue to experience widespread geographic inequalities in their chances 83 of survival. Sub-Saharan Africa is still the region with the highest child mortality rate in the 
world. The region had an average child mortality rate of 76 deaths per 1000 live births in 2019 . Over $80 \%$ of the 5.2 million child deaths occurred in sub-Saharan Africa and Central and Southern Asia. More than half of these deaths have occurred in sub-Saharan Africa. Three of the five countries (Ethiopia, Nigeria, and the Democratic Republic of Congo) in which half of the world's child deaths have occurred are in sub-Saharan Africa [4].

On the basis of the [5] estimates of national immunization coverage, Ethiopia is $5^{\text {th }}$ of the ten countries which account for the $62 \%$ of unprotected children following Nigeria, India, Democratic republic Congo and Pakistan.

The aim of this study is to understand the current status of vaccination coverage of children in Ethiopia and to reveal the various factors influencing the vaccination coverage in the country. The findings from this study would help the policymakers in understanding current status of vaccination coverage and the determinant of its coverage in the country and serve as an important input for any possible intervention aimed at improving the coverage in the country. This study covered children in the age range of 12- 23 months from all 9 regions and 2 city administrations of the country.

\section{Methods}

\section{Study Area}

Ethiopia is the oldest independent country, located in the North Eastern part of Africa, also known as the Horn of Africa, lies between 3 and 15 degrees north latitude and 33 and 48 degrees east longitude. Ethiopia is officially known as the Federal Democratic Republic of Ethiopia and is a landlocked country located in the Horn of Africa. It is the second-most populous nation in Africa, with $117,876,227$ populations according to united nation estimate of 2021 . The total surface area of Ethiopia is about 1.1 million square kilometers and bordered by Djibouti, Eritrea, the republic of the Sudan, the republic of the Southern Sudan, Kenya, and Somalia. At present Ethiopia is administratively structured in to ten regional states Tigray, Afar, Amhara, Somali, Benishangul-Gumuz, Southern Nations Nationalities and Peoples (SNNP), Gambela, Sidama and Harari and two city administrations, Dire Dawa and Addis Ababa. There are topographic-induced climatic variations broadly categorized into three: the "Kolla", or 
112 hot lowlands, below approximately 1,500 meters, the "Wayna Degas" at 1,500-2,400meters and

113 the "Dega" or cool temperate highlands above 2,400 meters [6].

\section{Data source and Description}

115 The data source for this study was secondary data from [6] which is the fourth Demographic and Health Survey (DHS) conducted in Ethiopia, following the 2000, 2005, and 2011 EDHS surveys.

117 The [6] provides valuable information on trends in key demographic and health indicators over 118 time. Information on vaccination coverage was obtained in three ways in the 2016 EDHS: written vaccination records (including the infant immunization card and other health cards), mothers' verbal reports, and health facility records. In the [6], for each child born in the 3 years before the survey, mothers was asked to provide information about the vaccinations that her child has received. Unlike the previous EDHS surveys, in the [6] a separate team visited the health facility to collect complementary vaccination records if the mother was not able to present

124 the infant immunization card and the child had visited a health facility. Consent was obtained from mothers prior to contacting the facilities and verifying child vaccination records.

The purpose of obtaining information at the health facility was to complement the information collected by mother's recall. The information collected through this [6] was intended to assist policy makers and program managers in evaluating and designing programs and strategies for improving the health of the country's population. Additionally, the [6] included a health facility component that recorded data on children's vaccinations, which were then combined with the household data on children's vaccinations. It was implemented by the Central Statistical Agency (CSA) at the request of the Ministry of Health $(\mathrm{MoH})$.

\section{Sampling Frame and Technique}

The sampling frame used for the [6] is the Ethiopia Population and Housing Census (PHC), which was conducted in 2007 by the Ethiopia Central Statistical Agency. The census frame is a complete list of 84,915 enumeration areas (EAs) created for the 2007 PHC. An EA is a geographic area covering on average 181 households. The sampling frame contains information about the EA location, type of residence (urban or rural), and estimated number of residential households. With the exception of EAs in six zones of the Somali region, each EA has accompanying cartographic materials. 
141 The sample for the 2016 EDHS was designed to provide estimates of key indicators for the 142 country as a whole, for urban and rural areas separately, and for each of the nine regions and the 143 two administrative cities. The 2016 EDHS sample was stratified and selected in two stages. Each

144 region was stratified into urban and rural areas, yielding 21 sampling strata. Samples of EAs 145 were selected independently in each stratum in two stages. Implicit stratification and 146 proportional allocation were achieved at each of the lower administrative levels by sorting the 147 sampling frame within each sampling stratum before sample selection, according to 148 administrative units in different levels, and by using a probability proportional to size selection at 149 the first stage of sampling.

150 In the first stage, a total of 645 Enumeration Areas (EAs) (202 in urban areas and 443 in rural 151 areas) were selected with probability proportional to EA size (based on the 2007 PHC) and with 152 independent selection in each sampling stratum.

\section{Inclusion and exclusion criteria}

Inclusion Criteria

155 As the study is related to children vaccination coverage in Ethiopia, children of age 12-23 156 month from all 9 regions and 2 city administrations were included.

157 Exclusion Criteria

158 In the 2016 EDHS, for each child born in the 3 years before the survey, mothers were asked to 159 provide information about the vaccinations her child has received.

160 The following children were excluded from the study:-

$161>$ Children less than the age of 11 months.

$162>$ Children greater than the age of 23 months.

163 Study variables

164 Dependent Variables

165 The dependent variable is the complete vaccination status of children aged 12-23 months. As 166 WHO recommended, basic childhood vaccines consists of polio, pentavalent (diphtheria, tetanus, 167 pertussis, Haemophilus influenza, and hepatitis B vaccine), measles, and Bacillus Calmette 
168

169

170

171

172

173

174

175

176

177

178

179

180

Guerin (BCG) that can prevent common childhood infections. Complete basic childhood vaccination achieved when the child received one dose of BCG vaccine, three doses of pentavalent vaccines, three doses of polio vaccines and one dose of measles vaccine. Additionally, three doses of the pneumococcal vaccine (PCV) and two doses of the rotavirus vaccine (RV) introduced by the government of Ethiopia.

The child who received all the above doses of each vaccine was categorized as "completely vaccinated". While those who failed to take the recommended doses of vaccine were categorized as "incompletely vaccinated".

A random variable $Y_{i}$ represents the $\mathrm{i}^{\text {th }}$ child response variable with two categories.

$Y_{i j}=\left\{\begin{array}{l}1, \text { if the } \mathrm{i}^{\text {th }} \text { child in } \mathrm{j}^{\text {th }} \text { region is completely vaccinated } \\ 0, \text { if the } \mathrm{i}^{\text {th }} \text { child in } \mathrm{j}^{\text {th }} \text { region is incompletely vaccinated }\end{array}\right.$

With $P_{i j}=P\left(Y_{i j}=1 / X_{i j}\right)$ is the probability $i^{t h}$ child in region $j$ fully vaccinated, $X_{i j}$ is the observed characteristic of the $i^{\text {th }}$ child in the $j^{\text {th }}$ region and $1-P_{i j}=P\left(\mathrm{Y}_{\mathrm{ij}}=0 / X_{i j}\right)$ is the probability of incomplete vaccination coverage for $i^{\text {th }}$ child in region $j$.

\section{Independent Variables}

Vaccination level of children is influenced by socio-economic and demographic factors. These includes maternal educational level, marital status, wealth Index, age of mother, place of delivery, child lives with who (care giver), women Occupation, ANC follow up, baby Post natal care, place of residence, sex of the child, type of pregnancy, field worker visit and husband educational level. These variables are supported with literatures.

\section{Method of Statistical Data Analysis}

\section{Multilevel Linear Models}

When the data was collected in hierarchical or clustered structures the suitable model is multilevel models. Multilevel models are used to account for the correlation of observations within a given group by incorporating group specific random effects. These random effects can be nested (individuals nested in regions, with random effects at the women and region levels) [7].The dependent variable must be examined at the lowest and highest level of analysis. 


\section{Bayesian Multilevel Logistic Regression Model}

\section{Two- level model}

This model considers the lack of independence across levels of nested data (i.e., individuals nested within regions). All experimental units are assumed to be independent which means that any variable which affects level of vaccination have the same effect in all regions, but these models are aiming at examining whether the effect of variables vary from region to region. The probability of 'success' or 'failure' is the same for all individuals in the group [7].

The random variable $Y_{i j}$ has a Bernoulli distribution is one of the standard assumption of the model. Similar to logistic regression the $p_{i j}$ is modeled using the link function logit. The two level models are given by:

$$
\operatorname{logit}\left(p_{i j}\right)=\log \left(\frac{p_{i j}}{1-p_{i j}}\right)=\beta_{o}+\sum_{h=1}^{k} \beta_{h j} x_{h i j}+U_{0 j}+\sum_{h=1}^{k} U_{h j} x_{h i j}
$$

Where $X_{h i j}=\left(X_{1 i j}, X_{2 i j}, \ldots \ldots, X_{k i j}\right)$ represents the first and the second level of covariates for $\mathrm{k}$ variables, $\beta=\left(\beta_{0}, \beta_{1}, \ldots \ldots \ldots \ldots, \beta_{k}\right)$ are regression parameter coefficients, and $U_{0 j}, U_{1 j}, \ldots \ldots \ldots \ldots U_{k j}$ are the random cluster effects of model parameter at higher levels with the assumption that follows normal distribution with mean zero and variance $\sigma_{u}^{2}$. Therefore, conditional on $U_{0 j}, U_{1 j}, \ldots \ldots \ldots U_{k j}$, the $Y_{i j}$ can be assumed to be independently distributed as Bernoulli random variables [7].

\section{Bayesian Multilevel Analysis of Empty Models}

The null two-level model or empty two-level model for a dichotomous outcome variable indicates to a population of groups (level-2 units) and dictates the probability distribution for group dependent probabilities $p_{j}$ in $Y_{i j}=p_{j}+\varepsilon_{i j}$ without taking further explanatory variables into account. Thus, in the population groups, the log-odds have a normal distribution, which is given by:-

$$
\mathrm{f}\left(p_{j}\right)=\beta_{0}+u_{0 j}
$$


218 Where $p_{j}=\frac{e^{\beta_{0}+u_{0 j}}}{1+e^{\beta_{0}+u_{0 j}}}, \beta_{0}$ is the population average of the transformed probabilities and $u_{0 j}$ are 219 regional level random effects that are i.i.d. normally distributed with zero means and constant 220 variances $\sigma_{u}^{2}$. This model does not constitute a different parameter for the level-one variance, 221 because the level-one error variance of the $Y_{i j}$ follows Bernoulli distribution straight from the 222 success probability, as indicated by $\operatorname{Var}\left(\epsilon_{i j}\right)=p_{j}\left(1-p_{j}\right)[7]$.

223 The likelihood function of empty model is

$$
\mathrm{L}\left(p_{j} / y_{i j}\right)=\prod_{j}\left(p_{i j}\right)^{y i j}\left(1-p_{j}\right)^{1-y i j}
$$

\section{Prior distribution}

226 The prior distributions are default non-informative uniform distribution for the intercept $\beta_{0}$ and 227 gamma for the random part $\sigma_{u 0}^{2}$ of the model. For the parameters. It is written as; $\mathrm{P}\left(\beta_{0}\right) \propto 1$ and $228 \mathrm{P}\left(\sigma_{u 0}^{-2}\right) \propto$ gamma $(\alpha, \beta)$ where $\alpha$ and $\beta$ are scale and shape parameters which are fixed constants 229 [7].

\section{Posterior Distribution}

231 From the posterior distribution $\mathrm{P}\left(\sigma_{u 0}^{2}, \beta_{0} / y_{i j}\right)$, we can estimate random parameters by using 232 MCMC methods.

233 The full conditional distribution for parameter $\beta_{0}$ is given by,

$$
\mathrm{P}\left(\beta_{0} / \sigma_{u 0}^{2}, y_{i j}\right) \propto \prod_{i j}\left(\frac{e^{\beta_{0}+u_{0 j}}}{1+e^{\beta_{0}+u_{0 j}}}\right)^{y i j}\left(\frac{1}{1+e^{\beta_{0}+u_{0 j}}}\right)^{1-y i j}
$$

235 The full conditional distribution for parameter $\sigma_{u 0}^{2}$ is given by,

$$
\mathrm{P}\left(\sigma_{u 0}^{2} / \beta_{0}, y_{i j}\right) \propto \operatorname{gamma}(\mathrm{N} / 2+\mathrm{N}(\alpha-1), \mathrm{N} \beta)
$$

237 Where $\mathrm{N}$ is the total number of individual respondents interviewed in all regions of the country 238 that is calculated from $\sum n j$ and the scale and shape parameters are $\alpha$ and $\beta$ respectively which 239 are both fixed constants [7]. 


\section{Variance Components Model}

242 The variance components model has the form;

$$
Y_{i j}=\beta_{0}+u_{0 j}+\epsilon_{i j}
$$

244 Where the vaccination status of the $i^{\text {th }}$ women in $j^{\text {th }}$ region is $Y_{i j}$, the regional level random 245 effects $u_{0 j}$ 's are i.i.d. normally distributed with mean zero and constant variances $\sigma_{u}^{2}$. The error 246 terms $\epsilon_{i j}$ are i.i.d. normally distributed with zero means and constant variances $\sigma_{e}^{2}$ and $\beta_{0}$ is the 247 overall mean.

248 The total variance is partitioned into two the region and children levels variances, representing 249 between and within region variability's in the utilization of antenatal care services [8]. The 250 interclass correlation (ICC) that measures correlation between observations within cluster 251 (region) is given by:

253 where $\sigma_{u}^{2}$ is the 'between-region' variance and $\sigma_{\varepsilon}^{2}$ is the 'between-children' variance .

\section{Bayesian Multilevel Analysis of Random Intercept Models}

255 In this model, the intercept is the only random effect that differ with respect to the average value 256 of the response variable, but the relationship between explanatory and response variables cannot 257 differ between groups. It shows the heterogeneity between groups in the overall response [7].

258 There is an assumption that there are variables which are potential explanation for observed 259 success or failure. These variables are denoted by:-

$260 X_{h}=\left\{X_{h i j}, \mathrm{i}=1,2, \ldots . n_{j}, \mathrm{~h}=1,2, \ldots \ldots, \mathrm{k}\right.$ and $\mathrm{j}=1,2, \ldots \ldots, \mathrm{N}, \mathrm{k}$ is the number of predictors $\}$

261 The logit of $p_{i j}$ which is called log odds is the sum of a linear function of the explanatory 262 variables and a random group-dependent deviation $u_{0 j}$.

$$
\operatorname{logit}\left(p_{i j}\right)=\beta_{0}+\sum_{h=1}^{k} \beta_{h} x_{h i j}+u_{0 j}
$$


264 Where the fixed part is $\beta_{0}+\sum_{h=1}^{k} \beta_{h} x_{h i j}$ and the remaining part $u_{0 j}$ is the random part of the model [9] and the success probability is

$$
p_{i j}=\frac{e^{\beta_{0}+\sum_{h=1}^{k} \beta_{h} x_{h i j}+u_{0 j}}}{1+e^{\beta_{0}+\sum_{h=1}^{k} \beta_{h} x_{h i j}+u_{0 j}}}
$$

\section{Bayesian Multilevel Analysis of Random Coefficients Models}

268

269

270

271

272

273

274

275

276

277

278

279

280

281

In this model, the coefficients of lower-level predictors are modeled. It indicates the unobserved heterogeneity in the effects of explanatory variables on the response variable [7].

We represent the variables that are potential explanations for the observed outcomes variables by $X_{h}=\left\{X_{h i j,} \mathrm{i}=1,2, \ldots . n_{j}, \mathrm{~h}=1,2, \ldots \ldots, \mathrm{k}\right.$ and $\left.\mathrm{j}=1,2, \ldots \ldots, \mathrm{N},\right\}$. The success probability is not constant for all individuals in a given group, as some or all of these variables could be level-one variable. Therefore, the success probability depends on the individual as well as the group, and is symbolized by $p_{i j}$.

$$
\operatorname{logit}\left(p_{i j}\right)=\log \left(\frac{p_{i j}}{1-p_{i j}}\right)=\beta_{0}+\sum_{h=1}^{k} \beta_{h j} x_{h i j}+u_{0 j}+\sum_{h=1}^{k} u_{h j} x_{h i j}
$$

In the above model, the part $\beta_{0}+\sum_{h=1}^{k} \beta_{h j} x_{h i j}$ is the fixed part and $u_{0 j}+\sum_{h=1}^{k} u_{h j} x_{h i j}$ is the random part [8]. The remaining part $\sum_{h=1}^{k} u_{h j} x_{h i j}$ can be considered as a random interaction between group and the explanatory variables. In other words, the groups are characterized by two random effects: their intercept and slopes [7].

\section{Prior Distribution}

Let us represent the parameters $\beta_{0}, \beta_{1}, \ldots \ldots \ldots \beta_{k}$ and $\Omega_{u}$ as prior distribution as follows.

$\mathrm{P}\left(\beta_{0}\right) \propto 1, \mathrm{P}\left(\beta_{1}\right), \ldots \ldots \ldots \ldots \mathrm{P}\left(\beta_{k}\right) \propto 1$ and $\mathrm{p}\left(\Omega_{u}\right) \sim$ inverse-Wishart ${ }_{\mathrm{k}}\left(s_{u}, \mathrm{~h}\right)$ distribution. Where $\Omega_{u}$ is the variance covariance matrices, $s_{u}$ is an estimate for the true value of $\Omega_{u}$, $\mathrm{k}$ is dimension of $\Omega_{u}$, that denotes the degree of freedom, so this prior is essentially as uninformative prior. In statistics, Wishart distribution is a generalization to multiple dimensions of the chi-squared distribution, or, in the case of non-integer degrees of freedom, of the gamma distribution [17]. Then $\Omega_{u}$ is positive definite with probability density function ; 


$$
\mathrm{f}\left(\Omega_{u}\right)=\frac{|s|^{\frac{h}{2}}}{|\Omega|^{\frac{h+p+2}{2}} 2^{\frac{h k}{2}} \operatorname{gamma}(k)^{\frac{h}{2}}} \exp \left\{-\frac{1}{2} \operatorname{tr}\left(s^{-1} \Omega\right)\right\}
$$

The posterior distribution for the parameters $\beta_{0}, \beta_{1}, \beta_{2}, \ldots \ldots \ldots \ldots, \beta_{k}$ is given by:-

$$
\mathrm{P}\left(\beta_{\hat{h}} / \Omega_{u}, y_{i j}\right) \propto \prod_{i j}\left(\left(p_{i j}\right)^{y i j}\left(1-p_{i j}\right)^{1-y i j}\right)
$$

Where $\hat{h}=0,1,2, \ldots \ldots, \mathrm{k}$. The full conditional distribution for the parameter $\Omega_{\boldsymbol{u}}$ is given by;

$$
\left.\mathrm{f}\left(\Omega_{u} / \beta_{\widehat{h}}, y_{i j}\right) \propto \mathrm{p}\left(y_{i j} / \Omega_{u}, \beta_{h}\right)\right)\left(\mathrm{f}\left(\Omega_{u}\right)\right)
$$

\section{Estimation Techniques for MCMC}

The software MLwiN, a specialized program for performing multilevel analysis was used to run the Bayesian estimation. The general simulation method Markov chain Monte Carlo (MCMC)is used for sampling from posterior distributions and computing posterior quantities of interest [7].

The Deviance Information Criterion (DIC) which is hierarchical modeling generalization of the AIC is used for model selection. Assessing goodness of fit involves examining how close the values are predicted by the model with that of observed values [9]. The comparison of observed to predicted values using the likelihood function is based on the statistic called deviance.

$$
\mathrm{D}=-2 \sum\left[y_{i} \log \left(p_{i}\right)+\left(1-y_{i}\right) \log \left(1-p_{i)}\right]\right.
$$

Where the predicted value for observation $\mathrm{i}$ is $p_{i}$.The rule is, the larger deviance, the poorer the fitness of the model [7].

\section{Missing Data}

Missing data is a problem since nearly all standard statistical methods presume complete information for all the variables is included in the analysis. A relatively few absent observations on some variables can dramatically shrink the sample size. As a result, the precision of confidence intervals is harmed, statistical power weakens and the parameter estimates may be biased. Appropriately dealing with missing can be challenging as it requires a careful examination of the data to identify the type and pattern of missing data, and also a clear understanding of how the different imputation methods work. 
312 Several different methods and strategies are available to handle missing data. First, the method

313 should yield unbiased estimates of a variety of different parameters. Second, the method should

314 include a way to assess the uncertainty about the parameter estimates, and third, the method

315 should have good statistical power. It is further good to remember that the goal of any missing

316 data procedure is not to recreate the missing observations but rather to retain the characteristics

317 of the data and the associations between variables such that valid and efficient inferences can be 318 made [10].

319 Multiple imputations are the popular method for handling missing values in a data. The 320 advantage of the method is that once the imputed data set have been generated, the analysis can 321 be carried out using procedures in virtually any statistical package, which makes the analysis 322 simple. Also, standard error or p-value obtained from MI is generally valid because they 323 incorporate uncertainty due to missing values. MI can be highly efficient even if the number of 324 imputations is relatively small, especially when between-imputation variance is not too large. 325 The multiple imputation inference involves three distinct phases [10].

326 As a rule of thumb, if less than $5 \%$ of the observation are missing, the missing data can be 327 simply be deleted without any significant ramifications [10]. For this particular study, the 328 percentage of mission observation is less than 5\%. However, if more than 5\% of the data is 329 missing, deleting the missing data will result in a reduced sample size and an increased standard 330 error of the parameter estimate. In this case it is strongly suggested to use imputation of the 331 mean, mode or median or multiple imputations, to fill in the missing data.

\section{Result and Discussion}

\section{Descriptive Statistics}

334 The results displayed in Table 1 below shows the percentages and counts of vaccination services 335 with respect to socioeconomic and demographic variables. All computations were conducted in 336 Statistical Package for Social Sciences (SPSS) version 20.0 and MLwiN version 2.36. Out of the 3371929 children who received vaccination service, $699(36.2 \%)$ children were fully vaccinated 338 (completely vaccinated), while 1230 (63.8\%) children were not fully vaccinated (incompletely 339 vaccinated) at the time of data collection. 
Table 1: Chi-square analysis output depicting the relationship between vaccination status and some

341 socio-economic and demographic variables

\begin{tabular}{|c|c|c|c|c|c|c|c|c|}
\hline \multirow[t]{2}{*}{ Variable } & \multirow[t]{2}{*}{ Category } & \multirow[t]{2}{*}{$\mathrm{N}$} & \multirow{2}{*}{$\begin{array}{l}\text { Percent } \\
(\%)\end{array}$} & \multicolumn{2}{|c|}{ Vaccination status } & \multirow[t]{2}{*}{ Df } & \multirow{2}{*}{$\begin{array}{l}\text { Chi- } \\
\text { square }\end{array}$} & \multirow[t]{2}{*}{ p-value } \\
\hline & & & & Complete (\%) & $\begin{array}{l}\text { Incomplete } \\
(\%)\end{array}$ & & & \\
\hline \multirow[t]{12}{*}{ Region } & Tigray & 216 & 11.2 & $114(52.78)$ & $102(47.22)$ & 10 & 243.41 & $\mathrm{P} \leq 0.001$ \\
\hline & Afar & 171 & 8.9 & $11(6.43)$ & $160(93.57)$ & & & \\
\hline & Amhara & 178 & 9.2 & $70(39.32)$ & $108(60.68)$ & & & \\
\hline & Oromia & 287 & 14.9 & $92(32.05)$ & $195(67.95)$ & & & \\
\hline & Somalia & 223 & 11.6 & $41(18.38)$ & $182(81.62)$ & & & \\
\hline & Benishangul- & 156 & 8.1 & $66(42.31)$ & $90(57.69)$ & & & \\
\hline & Gumuz & & & & & & & \\
\hline & SNNPR & 231 & 12.0 & $94(40.69)$ & $137(59.31)$ & & & \\
\hline & Gambella & 138 & 7.1 & $28(20.28)$ & $110(79.72)$ & & & \\
\hline & Harari & 117 & 6.1 & $39(33.33)$ & $78(66.67)$ & & & \\
\hline & Addis Abeba & 102 & 5.3 & $78(76.47)$ & $24(23.53)$ & & & \\
\hline & Dire dawa & 110 & 5.7 & $66(60)$ & $44(40)$ & & & \\
\hline \multirow[t]{2}{*}{ Residence } & Urban & 409 & 21.2 & $236(57.7)$ & $173(42.3)$ & 1 & 103.50 & $\mathrm{P} \leq 0.001$ \\
\hline & Rural & 152 & 78.8 & $463(30.5)$ & $1057(69.5)$ & & & \\
\hline \multirow{4}{*}{$\begin{array}{l}\text { Maternal } \\
\text { Educational } \\
\text { level }\end{array}$} & No education & 1170 & 60.65 & $312(26.67)$ & $858(73.33)$ & 3 & 136.75 & $\mathrm{P} \leq 0.001$ \\
\hline & Primary & 508 & 26.33 & $233(45.86)$ & $275(54.14)$ & & & \\
\hline & Secondary & 162 & 8.4 & $95(58.64)$ & $67(41.36)$ & & & \\
\hline & Higher & 89 & 4.6 & $59(66.29)$ & $30(33.71)$ & & & \\
\hline Wealth index & Poorest & 664 & 34.42 & $117(17.62)$ & $547(82.38)$ & 4 & 180.56 & $\mathrm{P} \leq 0.001$ \\
\hline
\end{tabular}




\begin{tabular}{|c|c|c|c|c|c|c|c|c|}
\hline & Poorer & 373 & 19.33 & $140(37.53)$ & $233(62.47)$ & & & \\
\hline & Middle & 339 & 17.57 & $144(42.48)$ & $195(57.52)$ & & & \\
\hline & Richer & 287 & 14.87 & $151(52.61)$ & $136(47.39)$ & & & \\
\hline & Richest & 266 & 13.78 & $147(55.26)$ & $119(44.74)$ & & & \\
\hline \multirow{2}{*}{$\begin{array}{l}\text { Field worker } \\
\text { visit }\end{array}$} & No & 129 & 66.93 & $308(23.85)$ & $983(76.15)$ & 1 & 258.87 & $\mathrm{P} \leq 0.001$ \\
\hline & Yes & 638 & 33.07 & $391(61.29)$ & $247(38.71)$ & & & \\
\hline \multirow{3}{*}{$\begin{array}{l}\text { Wanted last } \\
\text { child }\end{array}$} & Wanted & 154 & 80.14 & $544(35.18)$ & $1002(64.81)$ & 2 & 18.26 & $\mathrm{P} \leq 0.001$ \\
\hline & Wanted later & 271 & 14.05 & $126(46.49)$ & $145(53.51)$ & & & \\
\hline & $\begin{array}{l}\text { Wanted no } \\
\text { more }\end{array}$ & 112 & 5.8 & $29(25.89)$ & $83(74.11)$ & & & \\
\hline \multirow{2}{*}{$\begin{array}{l}\text { Sex of the } \\
\text { child }\end{array}$} & Male & 946 & 49.04 & $318(33.62)$ & $628(66.38)$ & 1 & 5.52 & 0.019 \\
\hline & Female & 983 & 50.96 & $381(38.76)$ & $602(61.24)$ & & & \\
\hline \multirow{2}{*}{$\begin{array}{l}\text { Mother } \\
\text { occupation }\end{array}$} & No work & 111 & 58 & $364(32.53)$ & $755(67.47)$ & 4 & 19.04 & $\mathrm{P} \leq 0.001$ \\
\hline & Gov'tal and & 257 & 13.32 & $115(44.75)$ & $142(55.25)$ & & & \\
\hline \multirow[t]{4}{*}{ type } & skill & & & & & & & \\
\hline & Agricultural & 386 & 20.01 & $149(38.60)$ & $237(61.40)$ & & & \\
\hline & $\begin{array}{l}\text { Household and } \\
\text { domestic }\end{array}$ & 35 & 1.81 & $13(37.14)$ & $22(62.86)$ & & & \\
\hline & Other & 132 & 6.8 & $58(43.94)$ & $74(56.06)$ & & & \\
\hline \multirow{2}{*}{$\begin{array}{l}\text { ANC follow } \\
\text { up }\end{array}$} & Yes & 855 & 44.32 & $478(55.91)$ & $377(44.09)$ & 1 & 257.15 & $\mathrm{P} \leq 0.001$ \\
\hline & No & 107 & 55.68 & $221(20.58)$ & $853(79.42)$ & & & \\
\hline \multirow{2}{*}{$\begin{array}{l}\text { Place of } \\
\text { delivery }\end{array}$} & Home & 115 & 59.82 & $373(32.32)$ & $781(67.68)$ & 1 & 19.04 & $\mathrm{P} \leq 0.001$ \\
\hline & Health facility & 775 & 40.18 & $326(42.06)$ & $449(57.94)$ & & & \\
\hline Baby PNC & No & 168 & 87.24 & $577(34.28)$ & $1106(65.72)$ & 1 & 21.77 & $\mathrm{P} \leq 0.001$ \\
\hline
\end{tabular}




\begin{tabular}{|c|c|c|c|c|c|c|c|c|}
\hline check up & Yes & 246 & 12.76 & $122(49.59)$ & $124(50.41)$ & & & \\
\hline Husband/ & No education & 891 & 46.19 & $284(31.87)$ & $607(68.13)$ & 4 & 30.54 & $\mathrm{P} \leq 0.001$ \\
\hline $\begin{array}{l}\text { partner } \\
\text { education }\end{array}$ & Primary & 620 & 32.14 & $226(36.45)$ & $394(63.55)$ & & & \\
\hline level & Secondary & 224 & 11.61 & $87(38.84)$ & $137(61.16)$ & & & \\
\hline & Higher & 189 & 9.79 & $99(52.38)$ & $90(47.62)$ & & & \\
\hline
\end{tabular}

343 On the basis of $\mathrm{p}$-value in the table 1 above, the vaccination coverage of children is associated 344 with region, residence type, educational level of the mother, wealth index, field worker visit, sex 345 of the child, wanted child, mother occupation, antenatal care service follow up, place of delivery, 346 baby postnatal care, husband/partner educational level.

347 Bayesian Multilevel Logistic Regression Analysis output

348 In the multilevel analysis, a two-level structure is used with regions as the second-level units and 349 children as the first level units. The expectation is that there would be differences in the level of 350 vaccination coverage among regions. The nesting structure is children within regions with a total 351 of 1929 children.

\section{Model Comparison}

353 Table 2: Bayesian Deviance Information Criteria

\begin{tabular}{ccccl}
\hline $\bar{D}$ & $\mathrm{D}(\bar{\theta})$ & $\mathrm{pD}$ & $\mathrm{DIC}$ & Model \\
& & & & \\
\hline 2037.71 & 1778.89 & 258.82 & 2296.53 & Bayesian multilevel null model \\
1767.29 & 5703.45 & 207.53 & 1974.82 & Random intercept model \\
1514.14 & 5606.13 & 340.11 & 1854.26 & Random Coefficient model \\
\hline
\end{tabular}


355 From Table 2, the measure $\bar{D}$ indicates the average deviance from the complete set of iterations.

$356 \mathrm{D}(\bar{\theta})$ indicates the deviance at the expected value of the unknown parameters and $\mathrm{pD}$ (the 357 estimated degrees of freedom consumed in the fit or it is the difference between the average 358 deviance from the complete set of iterations and the deviance at the expected value of the 359 unknown parameters. Also the deviance information criterion is the sum of $\bar{D}$ and $\mathrm{pD}$. For 360 comparing models, we use the DIC (Deviance Information Criteria). The random intercept model 361 reduced by 321.71 than the null model. Thus, the random intercept model with the fixed 362 explanatory variables shows a highly significant improvement suggesting that it is better than the 363 null model and the DIC diagnostics of random coefficient model is reduced by 120.56 and 364442.27 from the random intercept model with the fixed explanatory variables and null model 365 respectively. Therefore this smallest value of DIC criteria indicates that Bayesian multilevel 366 logistic regression for random coefficient model is the most significant model as compared to the 367 null and random intercept model with the fixed explanatory variables. Therefore, this study 368 employs the Bayesian multilevel logistic regression for random coefficient model.

\section{Bayesian Multilevel Logistic Regression Analysis using Empty Model}

370 The empty (null) model in table 3 contains no explanatory variables and it can be considered as a 371 parametric version of assessing heterogeneity of children vaccination coverage among regions.

372 Here also non informative priors were used for the fixed intercept and for the variance. The 373 following table 3 value was calculated from the posterior distribution by storing 50000 actual 374 iterations and from which the overall mean of vaccination coverage is estimated at $\beta_{0}=-0.660$ 375 and significant at $5 \%$ level of significance.

Table 3: Bayesian Multilevel Logistic Regression Empty Model

\begin{tabular}{lllll}
\hline Model & Coefficient & Standard error & Z-value & P-value \\
\hline Fixed intercept $\left(\beta_{0}\right)$ & -0.660 & 0.084 & -7.86 & $\mathrm{P} \leq 0.001$ \\
Random effect & & & \\
Variance $=\sigma_{u 0}^{2}$ & 1.739 & 0.309 & 5.63 & \\
ICC & 0.3458 & & \\
\hline
\end{tabular}


Using table 3 above, it is possible to test the following hypothesis:

Ho: there is no regional variation on vaccination coverage of the children.

$\mathrm{H} 1$ : there is a regional variation in the coverage of vaccination.

By using the $\mathrm{p}$-value from the above table, which is $\mathrm{P} \leq 0.001$, we reject the null hypothesis and conclude that there is a regional variation (heterogeneity) on vaccination coverage of the children.

The variances $\sigma_{\varepsilon}^{2}$ and $\sigma_{u}^{2}$ in table 3 estimate the variation among individual child and among regions of the country. The individual (level-1) variance was fixed to $\pi^{2} / 3=3.29$ for the logit model. Also, to examine how much variation in the coverage of vaccination of child was attributable to the region level factors, it is useful to see the intra-region correlation coefficient ICC $=\frac{\sigma_{u}^{2}}{\sigma_{u}^{2}+\sigma_{\varepsilon}^{2}}=1.739 / 1.739+3.29=0.3458$, which measures the proportion of variance of complete coverage of vaccination that is between regions, not within regions. The intra-region correlation coefficient (ICC) in the intercept-only model is 0.3458 which is significant at a $5 \%$ level of significance. This means that $34.58 \%$ of the variation in coverage of vaccination exists between regions, whereas the remaining $65.42 \%$ attributable to an individual (children) level, i.e. within region variation.

\section{Bayesian Multilevel Logistic Regression Random Intercept Model}

To identify the effect of explanatory variables, a Bayesian multilevel binary logistic model with random intercept and fixed explanatory variables was estimated and displayed in table 4 below. The following table is the output from MLwin 2.36 for the Bayesian multilevel random intercept model.

\section{Table 4: Results of Random Intercept Bayesian Multilevel Logistic Regression Model}

\begin{tabular}{|c|c|c|c|c|c|}
\hline Variable & Category & Coefficient & Standard error & Z-value & $\mathrm{P}$-value \\
\hline Fixed & Intercept & -2.305 & 0.819 & -2.81 & $\mathrm{P} \leq 0.001$ \\
\hline \multirow[t]{2}{*}{ Residency } & Rural & -1.216 & 0.221 & -5.50 & $\mathrm{P} \leq 0.001$ \\
\hline & Urban (ref) & & & & \\
\hline Age of the & $20-24$ & -0.012 & 0.303 & -0.04 & 0.497 \\
\hline
\end{tabular}




\begin{tabular}{|c|c|c|c|c|c|}
\hline \multirow[t]{6}{*}{ mother } & $25-29$ & 0.150 & 0.323 & 0.46 & 0.326 \\
\hline & $30-34$ & 0.163 & 0.331 & 0.49 & 0.331 \\
\hline & $35-39$ & 0.393 & 0.367 & 1.07 & 0.173 \\
\hline & $40-44$ & 0.381 & 0.411 & 0.93 & 0.218 \\
\hline & $45-49$ & 0.709 & 0.698 & 0.99 & 0.158 \\
\hline & $15-19$ (ref) & & & & \\
\hline \multirow[t]{5}{*}{ Education } & Primary & 0.456 & 0.162 & 2.81 & 0.003 \\
\hline & Secondary & 0.637 & 0.269 & 3.94 & 0.009 \\
\hline & Higher & 0.224 & 0.372 & 0.60 & 0.274 \\
\hline & No education (ref & & & & \\
\hline & Poorer & 0.760 & 0.203 & 3.74 & $\mathrm{P} \leq 0.001$ \\
\hline \multirow[t]{4}{*}{ Wealth index } & Middle & 0.814 & 0.221 & 3.68 & $\mathrm{P} \leq 0.001$ \\
\hline & Richer & 1.372 & 0.232 & 5.92 & $\mathrm{P} \leq 0.001$ \\
\hline & Richest & 1.460 & 0.251 & 5.82 & $\mathrm{P} \leq 0.001$ \\
\hline & Poorest(ref) & & & & \\
\hline \multirow{3}{*}{$\begin{array}{l}\text { Sex of the } \\
\text { child }\end{array}$} & Female & 0.100 & 0.131 & 0.76 & 0.224 \\
\hline & Male (ref) & & & & \\
\hline & Wanted later & 0.465 & 0.183 & 2.54 & 0.007 \\
\hline \multirow[t]{2}{*}{ Child wanted } & Wanted no more & -0.713 & 0.321 & -2.22 & 0.016 \\
\hline & Wanted (ref) & & & & \\
\hline \multirow{2}{*}{$\begin{array}{l}\text { Field worker } \\
\text { visit }\end{array}$} & Yes & 1.685 & 0.140 & 12.04 & $\mathrm{P} \leq 0.001$ \\
\hline & No (ref) & & & & \\
\hline
\end{tabular}




\begin{tabular}{|c|c|c|c|c|c|}
\hline \multirow[t]{6}{*}{ Marital status } & Married & 0.406 & 0.751 & 0.54 & 0.318 \\
\hline & Living with partner & 0.707 & 1.024 & 0.69 & 0.252 \\
\hline & Widowed & 0.767 & 1.002 & 0.76 & 0.227 \\
\hline & Divorced & 0.336 & 0.831 & 0.40 & 0.362 \\
\hline & No longer living together & 0.429 & 1.111 & 0.39 & 0.355 \\
\hline & Never in union (ref) & & & & \\
\hline $\begin{array}{l}\text { Number of } \\
\text { child }\end{array}$ & & -0.030 & 0.107 & -0.28 & 0.390 \\
\hline \multirow[t]{2}{*}{ Baby PNC } & Yes & 0.579 & 0.191 & 3.03 & $\mathrm{P} \leq 0.001$ \\
\hline & No (ref) & & & & \\
\hline \multirow[t]{2}{*}{ ANC } & Yes & 1.777 & 0.141 & 12.6 & $\mathrm{P} \leq 0.001$ \\
\hline & No(ref) & & & & \\
\hline \multirow{2}{*}{$\begin{array}{l}\text { Place of } \\
\text { delivery }\end{array}$} & Health facility & -0.008 & 0.148 & -0.054 & 0.477 \\
\hline & Home (ref) & & & & \\
\hline Occupation & Gov'tal and skill & -0.160 & 0.207 & & 0.221 \\
\hline \multirow[t]{3}{*}{ type } & Agricultural & 0.425 & 0.179 & & 0.009 \\
\hline & House hold & -0.395 & 0.518 & & 0.225 \\
\hline & Other & 0.321 & 0.260 & & 0.110 \\
\hline
\end{tabular}

No work (ref)

Random effect

Variance

1.093

0.255

4.29

ICC

0.2493 
401 From table 4 above, we can understand that the covariates type of residency, women educational 402 level, wealth index, child wanted (desired), field worker visit in last 12 months, baby postnatal 403 care, antenatal care and women were also found to be significant and also contributing factors for 404 variation in children vaccination among the regions of Ethiopia.

405 Bayesian Multilevel Logistic Regression of Random Coefficient Model

406 The effect of level one covariate can be incorporated in the model through adding random 407 coefficients in front of some of the individual-level covariates of the model. In random intercept 408 model we allowed the intercept only vary across regions by fixing explanatory covariates, but the 409 relationship between explanatory and dependent variable cannot differ between groups. The 410 following table depicts some random coefficients and fixed explanatory variables which had 411 significant effect on the vaccination coverage of children in Ethiopia.

412 Table 5: Results of Random Coefficient Bayesian Multilevel Logistic Regression Model

\begin{tabular}{|c|c|c|c|c|c|c|}
\hline Fixed effect & Category & Estimate & Standard error & $\mathrm{Z}$ & p-value & OR \\
\hline Constant & & -2.377 & 0.325 & -7.31 & $\mathrm{P} \leq 0.001$ & 0.093 \\
\hline \multirow[t]{2}{*}{ Residency } & Rural & -1.451 & 0.247 & -5.87 & $\mathrm{P} \leq 0.001$ & 0.234 \\
\hline & Urban(ref) & & & & & \\
\hline \multirow{4}{*}{$\begin{array}{l}\text { Maternal } \\
\text { educational } \\
\text { level }\end{array}$} & Primary & 0.591 & 0.174 & 3.40 & $\mathrm{P} \leq 0.001$ & 1.81 \\
\hline & Secondary & 0.787 & 0.292 & 2.69 & 0.003 & 2.19 \\
\hline & Higher & 0.072 & 0.399 & 0.18 & 0.425 & 1.07 \\
\hline & No educatic & & & & & \\
\hline \multirow[t]{4}{*}{ Wealth index } & Poorer & 1.124 & 0.255 & 4.41 & $\mathrm{P} \leq 0.001$ & 3.077 \\
\hline & Middle & 1.188 & 0.243 & 4.89 & $\mathrm{P} \leq 0.001$ & 3.281 \\
\hline & Richer & 1.779 & 0.263 & 6.76 & $\mathrm{P} \leq 0.001$ & 5.924 \\
\hline & Richest & 1.959 & 0.307 & 6.38 & $\mathrm{P} \leq 0.001$ & 7.092 \\
\hline
\end{tabular}




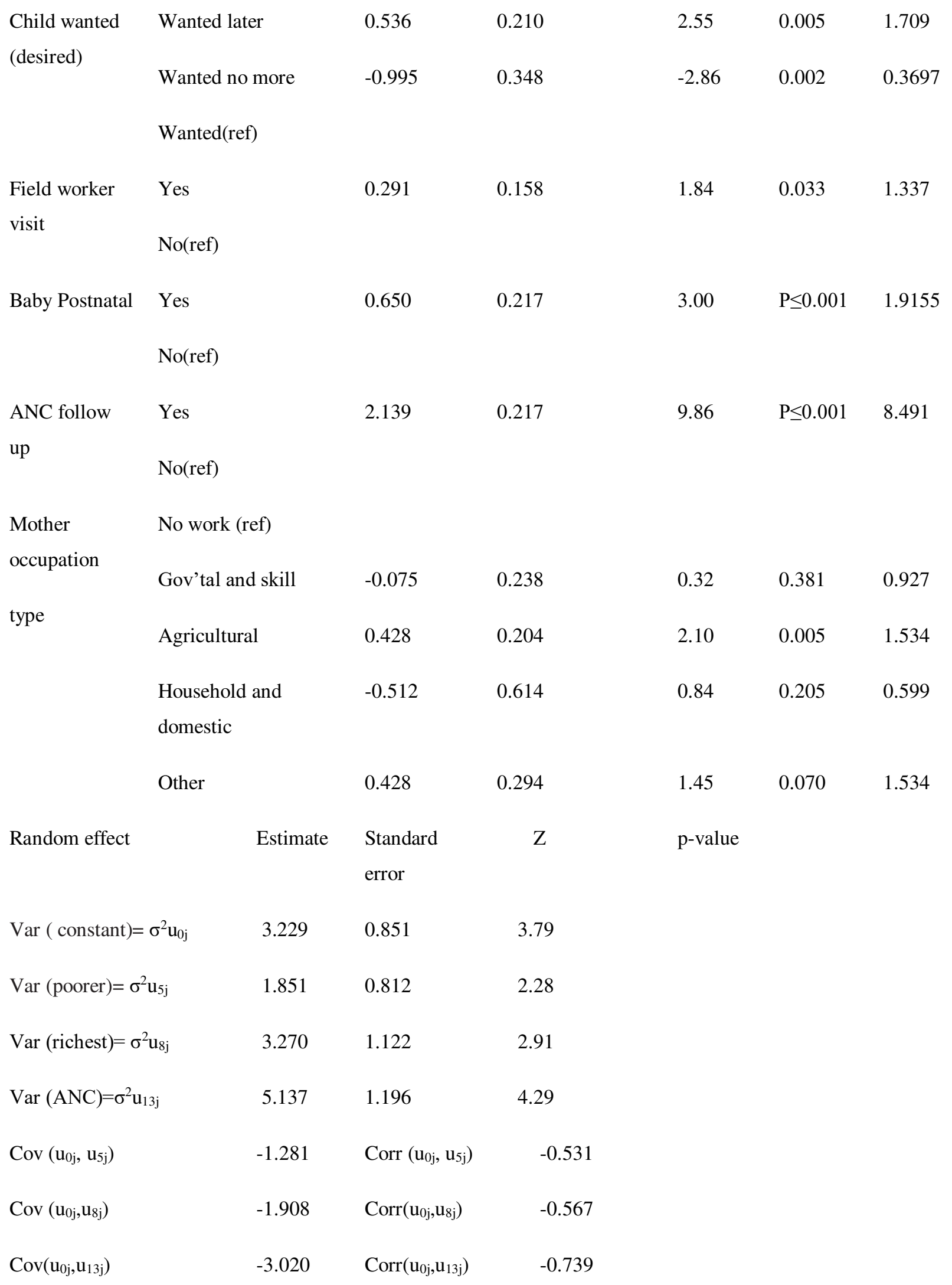




$\begin{array}{lcll}\operatorname{Cov}\left(\mathrm{u}_{5 \mathrm{j}}, \mathrm{u}_{8 \mathrm{j}}\right) & 0.761 & \operatorname{Corr}\left(\mathrm{u}_{5 \mathrm{j}}, \mathrm{u}_{8 \mathrm{j}}\right) & 0.315 \\ \operatorname{Cov}\left(\mathrm{u}_{5 \mathrm{j}}, \mathrm{u}_{13)}\right) & 0.881 & \operatorname{Corr}\left(\mathrm{u}_{5 \mathrm{j}}, \mathrm{u}_{13)}\right. & 0.291 \\ \operatorname{Cov}\left(\mathrm{u}_{8 \mathrm{j}}, \mathrm{u}_{13 \mathrm{j}}\right) & -0.192 & \operatorname{Corr}\left(\mathrm{u}_{8 \mathrm{j}}, \mathrm{u}_{13 \mathrm{j}}\right) & -0.063 \\ \operatorname{ICC} & 0.495 & & \end{array}$

413

414

415

As depicted in table 5 above, some of the independent variables were found to have significant effect on the vaccination coverage of children. These are women educational level, wealth index, type of residency, child wanted, field worker visit in last 12 month, baby postnatal care, antenatal care for current child and women occupation found to be significant at $5 \%$ significant level.

By adding level 1 predictors, the ICC depicted in table 5 increased to 0.4953 which indicates that roughly $49.53 \%$ of the total variability in child vaccination coverage is attributable to the random factor and region in random coefficient Bayesian multilevel binary logistic model. From table 5, the random coefficient estimates for intercepts and the slopes vary significantly at 5\% significance level, which implies that there is a considerable variation in the effects of wealth index and antenatal care; these variables differ significantly across the regions.

Using the OR value in table 5, the odds of vaccination coverage of children who dwell in rural areas was 0.234 indicating that a $76.6 \%$ decrease in coverage than children who dwell in urban.

Similarly in table 5 above, the odds of vaccination coverage among children having mother who had primary and secondary level of education was 1.81 and 2.19 respectively. This indicates that the odds of vaccination coverage of children of mothers with primary and secondary education are 1.81 and 2.19 times than children of mothers with no education at $5 \%$ level of significance and keeping all the other variables in the model constant.

As depicted in table 5 above, the odds of vaccination coverage of children in the poorer, middle, richer, richest wealth index category were 3.077, 3.281, 5.924 and 7.092 respectively which illustrates that the odds of vaccination coverage for children living in the poorer, middle, richer, richest wealth index category families are 3.077, 3.281, 5.924 and 7.092 times that of children living poorest household at 5\% level of significance and keeping all the other variables in the model constant. 
As provided in table 5 above, the odds of vaccination coverage among children with mothers who need pregnancy later and who wanted no more children were found to be 1.709 times and 0.3697 times than those children with mothers who need more children later respectively. Thus, children with mothers who do not need more children later are $63.03 \%$ less likely to cover vaccination than children with mothers who want to deliver more children later at $5 \%$ level of significance and keeping all the other variables in the model constant.

Similarly in table 5, the odds of vaccination coverage among children whose households had been visited by field worker in the last 12 month was found to be 1.337 compared to households who are not the visited by the field worker (ref). Therefore, children whose household are visited by field worker are $33.7 \%$ more likely to cover vaccination than those whose household was not visited by field worker at $5 \%$ level of significance and keeping all the other variables in the model constant.

As shown in table 5 above, the odds of vaccination coverage among children whose mother had attended postnatal care found 1.9155 indicating that they are $91.55 \%$ more likely to cover vaccination compared to children whose mother had not attended postnatal care services (ref) at $5 \%$ level of significance and keeping all the other variables in the model constant.

With regard to the variable ANC service shown in table 5, it has been found that the odds of vaccination coverage among children having mothers who attended antenatal care service was found 8.491 indicating that their odds is 8.491 times than that of children whose mother did not attend the ANC services at 5\% level of significance and keeping all the other variables in the model constant.

As it has been depicted in table 5, the odds of vaccination coverage among children having mother who had working in agriculture was found 1.534 compared to a women who had not working (ref). This shows that children with mother working in agriculture are $53.4 \%$ more likely to cover vaccination than children with mother who did not work in agriculture at $5 \%$ level of significance and keeping all the other variables in the model constant.

The estimated variance of intercept, slope of household wealth index and antenatal care follow up depicted in table 5 varies significantly. This showed that the factors household wealth index and antenatal care follow up have brought a variation in the vaccination coverage across regions 
of the country. Some of the variances of the interaction terms between intercepts and slopes of explanatory variables are also found significant.

In Table 5, the value of $\operatorname{var}\left(\mathrm{u}_{0 \mathrm{j}}\right), \operatorname{var}\left(\mathrm{u}_{5 \mathrm{j}}\right), \operatorname{var}\left(\mathrm{u}_{8 \mathrm{j}}\right)$ and $\operatorname{var}\left(\mathrm{u}_{13 \mathrm{j}}\right)$ are the estimated variance of intercept, slope of wealth index and antenatal care follow up. From the output, we can understand that there is a significant variation in the effects of these explanatory variables across the regions. Some of the variances of the interaction terms between intercepts and slopes of explanatory variables are also found significant. Interpretation of significant covariance terms can be easily made in terms of the correlation coefficients displayed in Table 5, and the correlation matrix contains the estimated correlation coefficients between random intercept and slopes (-0531, $-0.567,-0.739$ for poorer, richest and ANC follow up respectively) which are negatively correlated. The negative sign for the correlation between intercepts and slopes indicates that regions with higher intercepts tend to have on average lower slopes on the corresponding predictors.

\section{Discussion}

The purpose of this study was to understand the current status of complete vaccination coverage and associated factors among children in Ethiopia by using Bayesian Multilevel logistic regression Analysis. This study employed three multilevel logistic regression models for the response variable on the status of vaccination coverage. The Bayesian multilevel logistic regression empty model, Bayesian multilevel logistic regression random intercept, and Bayesian multilevel logistic regression for the random coefficient model were applied to explain the vaccination coverage among children of 12-23 month based on EDHS 2016 data.

Bayesian multilevel logistic regression random coefficient model was identified to be a better fit for the vaccination dataset. This model was also considered to be the best model to check the antenatal care service utilization in national level [12].

The upward trend in immunization coverage in recent years in Ethiopia was due to tremendous efforts of the government to realize the millennium development goal of reducing child mortality from vaccine preventable diseases. A study conducted by [13] in Afar, Somali and Tigray regions showed that the full vaccination coverage was $20.6 \%, 36.6 \%$ and $51 \%$ respectively. 
The place of residence was found to be significantly associated with the complete coverage of vaccination. This shows that children who were living in rural areas were less likely to complete vaccinations than children living in urban areas. This difference might be due to the fact that urban children are more accessible to health services and mothers of children have information and education about vaccination than women in the rural area as indicated by studies in $[13,14]$.

Children with mothers having primary and above education level are associated with an increased in full immunization. In other words, as mothers' level of education increase the probability of having a full immunization of children increased. This has been confirmed with the finding of [14].The importance of maternal education in children's health is universally recognized. Children of more educated mothers are more likely to be fully immunized.

Childhood immunization is also influenced by the household wealth index. A child from a family with poor wealth has a higher chance of being incompletely immunized. This might be associated with lack of money that results in poor health seeking behavior. Household income and wealth index influences the likelihood that children receive complete immunization. This result is similar to the results from $[14,15]$. This might be due to the indirect cost needed for travel to health facilities or time spent away from income generating activity to make it difficult for the poorest households to avail themselves of services that exist in the community [16].

The study also found that the type of pregnancy had significant effect on status of vaccine completion. On the basis of this study finding, a child that was born from mothers who wanted pregnancy was more likely to get complete vaccination. This finding matches with the finding from [1] that was conducted in Debre Markos town in Ethiopia.

The finding from this study also shows that the use of health care service (ANC and PNC) by mothers contributes to the improvement in the immunization status of children. Children with mothers who attended antenatal care have higher chance of being fully immunized compared to those whose mothers did not attend any antenatal care. Attending antenatal care may puts women in a better position of obtaining adequate information about routine immunization for children and other health facility services. Also, the processes undergone during antenatal care prepare a mother towards having positive inclination to health care utilization not only for themselves but also their children. According to the study maternal antenatal care follow up and baby post natal 
522 care are possible reasons for having better vaccination completion. Mothers who have more

523 frequent contact with health professionals seem to be more aware of their children's health as

524 they receive more information about immunization and child health. A study conducted by [4,

$52514,15,17]$ confirmed this truth.

526 This study depicts that worker visit of the family in the last 12 months was significantly 527 associated with the status of vaccine completion. This might be related with sharing of 528 information to mothers about immunization, including immunization schedules and side effects 529 during their visit. The finding is in conformance with a study done in Indonesia by [15].

530 The study also identified some socio-economic indicators of the region as predictors that affect 531 the status of vaccine completion. Variables like wealth index and antenatal care service 532 utilization are considered as region level factors. According to the random coefficient model, 533 these level-two variables explain $46.24 \%$ of the variation in vaccination completion status of 534 children in the country.

\section{Strength and limitations}

536 The statistical model used for the research need the application of various softwares. In this 537 regard, the contribution from authors is of high value and the findings of the research have less 538 standard error and high accuracy. Some important variables that would affect the vaccination 539 coverage have been missed from the dataset.

\section{Conclusion and Future Implication}

541 The study identified some socioeconomic and demographic factors that affect the status of 542 vaccine completion of children. Based on the findings, at the individual level, variables like 543 maternal (caregiver's) education level, household wealth index, mother occupation, ANC and 544 PNC service utilization were found to be the significant factors affecting complete coverage of children vaccination. These days, the number of women attending education is increasing. As a

546 result the coverage of vaccination would definitely increase in the near future. In this regard, all 547 stakeholders particularly the government should act to raise women's educational level and 548 promote the importance of using ANC and PNC to mothers of children. Moreover, the 549 government and other stakeholders working on health and related sectors should work on the 550 accessibility of health facilities around places where women lives so that they can save 
551 transportation cost and minimize waste of time. Finally, health extension workers should make

552 have a regular schedule to visit family having children taking vaccination.

\section{Abbreviations}

554 AIC Akakie information criteria

555 ANC Antenatal care

556 BCG Bacillus-Calmette-Guerin (Tuberculoses Vaccine)

557 BIC Bayesian information criterion

558 CSA Central Statistical Agency

559 DIC Deviance information criteria

560 DPT Diphtheria, pertussis, tetanus vaccine

561 EAs Enumeration areas

562 EDHS Ethiopia Demographic and Health Survey

563 EPHC Ethiopian Population and Housing Census

564 EPHI Ethiopia Public Health Institute

565 FMOH Federal Ministry of Health

566 GAVI Global Alliance for Vaccine and Immunization

567 GVAP Global vaccine action plan

568 GIVS Global Immunization Vision and Strategies

569 HepB Hepatitis B (vaccine)

570 Hib Haemophilus influenzae type B (vaccine)

571 ICC Interclass correlation

572 MDG Millennium Development Goal

573 MCMC Markov chain Monte Carlo

574 MCSE Monte Carlo standard error

575 ML Maximum Likelihood

576 MLE Maximum likelihood estimation

577 OR Odds Ratio 


\begin{tabular}{|c|c|c|}
\hline 578 & OPV & Oral Polio Vaccine \\
\hline 579 & PACF & Partial autocorrelation function \\
\hline 580 & PCV & Pneumococcal Conjugated Vaccine \\
\hline 581 & PNC & Post natal care \\
\hline 582 & RED & Reaching Every District \\
\hline 583 & RV & rotavirus vaccine \\
\hline 584 & SPSS & Statistical package for social science \\
\hline 585 & USAID & United States Agency for International Development \\
\hline 586 & VPD & Vaccine Preventable Diseases \\
\hline 587 & WHO & World Health Organization \\
\hline 588 & \multicolumn{2}{|c|}{ Acknowledgments } \\
\hline 589 & \multirow{2}{*}{\multicolumn{2}{|c|}{$\begin{array}{l}\text { The authors are grateful to the Central Statistical Agency of Ethiopia for providing the } 2016 \\
\text { DHS dataset of Ethiopia. }\end{array}$}} \\
\hline 590 & & \\
\hline 591 & \multicolumn{2}{|c|}{ Authors Contribution } \\
\hline 592 & \multirow{2}{*}{\multicolumn{2}{|c|}{$\begin{array}{l}\text { Both authors have contributed from the onset of the research, reviewing lit } \\
\text { verifying the data, analyzing, interpreting and write-up of the research. }\end{array}$}} \\
\hline 593 & & \\
\hline 594 & \multicolumn{2}{|c|}{ Funding } \\
\hline 595 & \multicolumn{2}{|c|}{ No funding was obtained for the whole work } \\
\hline 596 & \multicolumn{2}{|c|}{ Availability of data and materials } \\
\hline 597 & \multirow{3}{*}{\multicolumn{2}{|c|}{$\begin{array}{l}\text { In this study, we used the information from the fourth Demographic and Health Survey } \\
\text { conducted of Ethiopia from January 18, 2016, to June 27, } 2016 \text { by Central Statistical Agency } \\
\text { (CSA) focusing on all children in the age range 12-23 months. }\end{array}$}} \\
\hline 598 & & \\
\hline 599 & & \\
\hline 600 & \multicolumn{2}{|c|}{ Declaration } \\
\hline 601 & \multicolumn{2}{|c|}{ Ethics approval and consent to participate } \\
\hline 602 & \multicolumn{2}{|c|}{ Not applicable. } \\
\hline 603 & \multicolumn{2}{|c|}{ Consent for publication } \\
\hline 604 & \multicolumn{2}{|c|}{ Not applicable. } \\
\hline 605 & \multicolumn{2}{|c|}{ Competing interests } \\
\hline 06 & The aut & are that they have no competing \\
\hline
\end{tabular}




\section{References}

609 1. G. Tenaw and D. Abebe. "Vaccination Coverage and Associated Factors among Children

610 Aged 12-23 Months in Debre Markos Town, Amhara Regional State, Ethiopia”. Hindawi. 2017; 611 2017: 1-7.

612 2. WHO. "State of the world's vaccines and immunization" WHO Library Cataloguing-in613 Publication Data, 2009, Third edition.

614 3. Centers for Disease Control and Prevention, "Prevention: Vaccine," Centers for Disease 615 Control and Prevention, New York, NY, USA, 2017.

616 4. S.M. Fenta,., H.B. Biresaw, K.D. Fentaw. "Determinants of full childhood immunization 617 among children aged 12-23 months in sub-Saharan Africa: a multilevel analysis using 618 Demographic and Health Survey Data". Trop Med Health 49, 29 (2021). 619 https://doi.org/10.1186/s41182-021-00319-X.

620 5. WHO and UNICEF. "Ethiopia: Estimates of national immunization coverage: 2019 version" 621 July 6, 2020. World Health Organization Press, 2020.

622 6. Central Statistical Agency (CSA) [Ethiopia] and ICF, 2016 Ethiopia Demographic and Health 623 Survey Key Findings, CSA and ICF, Addis Ababa, Ethiopia, and Rockville, Maryland, USA, 6242017.

625

626 7. K.A. Cheru and D.T. Ehit. "Bayesian Multilevel Analysis of Utilization of Antenatal Care 627 Services in Ethiopia” Computational and Mathematical Methods in Medicine Volume 2020, 628 Article ID 8749753, 11 pages https://doi.org/10.1155/2020/8749753

629

630 8. M. H. R. Khan and J. E. H. Shaw, "Multilevel logistic regression analysis applied to binary 631 contraceptive prevalence data,” Journal of Data Science, vol. 9, no. 1, pp. 93-110, 2011.

632

633 9. T. A. B. Snijders and R. J. Bosker, "Multilevel analysis: an introduction to basic and 634 advanced multilevel modeling”, Sage Publishers, 2012. 
636

637

638

639

640

641

642

643

644

645

646

647

648

649

650

651

652

653

654

655

656

657

658

$659 \quad 16$

660

661

662

663

10. J. L. Schafer, and J. W. Graham. "Missing data: Our view of the state of the art”. Psychological Methods, 7(2), 147-177. https://doi.org/10.1037/1082-989X.7.2.147 , 2002.

11. Greenland S, Finkle WD. "A critical look at methods for handling missing covariates in epidemiological regression analyses”. Am J Epidemiol 1995;142:1255-64.

12. A. Cheru and T. Ehit. "Bayesian Multilevel Analysis of Utilization of Antenatal Care Services in Ethiopia" Computational and Mathematical Methods in Medicine, Volume 2020, Article ID 8749753, 11 pages

13. K.S Tamirat and M.M Sisay. "Full immunization coverage and its associated factors among children aged 12-23 months in Ethiopia: further analysis from the 2016 Ethiopia demographic and health survey". BMC Public Health. 2019; 19(1):1019. https://doi.org/10.1186/s12889-019$\underline{7356-2}$

14. S.T Adedokun and O.A Uthman, V.T Adekanmbi, C.S Wiysonge. "Incomplete childhood immunization in Nigeria: a multilevel analysis of individual and contextual factors". BMC Public Health. 2017;17(1):236

15. A. Holipah, A. Maharani, \& Y. Kuroda. "Determinants of immunization status among 12 - to 23-month-old children in Indonesia (2008-2013): a multilevel analysis". BMC Public Health 18, 288 (2018). https://doi.org/10.1186/s12889-018-5193-3.

16. A. Debie. "Complete vaccination service utilization inequalities among children aged 12-23 months in Ethiopia: a multivariate decomposition analyses”. Int J Equity Health. 2020;19(1):116

17. A.A Fatiregun, A.O Okoro. "Maternal determinants of complete child immunization among children aged 12-23 months in a southern district of Nigeria". Vaccine. 2012;30(4):730-6. 
664

665

666 